\title{
Wilson loop algebras and quantum K-theory for Grassmannians
}

\author{
Hans Jockers, ${ }^{a}$ Peter Mayr, ${ }^{b}$ Urmi Ninad ${ }^{a}$ and Alexander Tabler ${ }^{b}$ \\ ${ }^{a}$ Bethe Center for Theoretical Physics, Physikalisches Institut, Universität Bonn, \\ 53115 Bonn, Germany \\ ${ }^{b}$ Arnold Sommerfeld Center for Theoretical Physics, Ludwig-Maximilians-Universität, \\ 80333 Munich, Germany \\ E-mail: jockers@uni-bonn.de, mayr@physik.uni-muenchen.de, \\ urmi@th.physik.uni-bonn.de, tabler.alexander@physik.uni-muenchen.de
}

AbStRact: We study the algebra of Wilson line operators in three-dimensional $\mathcal{N}=2$ supersymmetric $\mathrm{U}(M)$ gauge theories with a Higgs phase related to a complex Grassmannian $\operatorname{Gr}(M, N)$, and its connection to K-theoretic Gromov-Witten invariants for $\operatorname{Gr}(M, N)$. For different Chern-Simons levels, the Wilson loop algebra realizes either the quantum cohomology of $\operatorname{Gr}(M, N)$, isomorphic to the Verlinde algebra for $\mathrm{U}(M)$, or the quantum K-theoretic ring of Schubert structure sheaves studied by mathematicians, or closely related algebras.

Keywords: Chern-Simons Theories, Supersymmetric Gauge Theory, Wilson, 't Hooft and Polyakov loops

ARXIV EPRINT: 1911.13286 


\section{Contents}

1 Introduction and outline 1

2 Generalized $I$-functions $\quad 2$

2.1 Generalized $I$-functions of $\operatorname{Gr}(M, N) \quad 2$

2.2 A window for the Chern-Simons levels 4

3 Algebra of difference operators 5

3.1 Wilson loops 5

3.2 Difference and differential equations for the $I$-function 7

4 Selected examples $\quad 9$

4.1 Quantum cohomology and factorized case 9

$\begin{array}{ll}4.2 \text { Chiral ring at level zero } & 10\end{array}$

$\begin{array}{lll}4.3 & \text { Difference equations at level zero } & 11\end{array}$

4.4 Perturbed theory for $\operatorname{Gr}(2,4) \quad 12$

A Perturbed structure constants for $\mathbb{P}^{5}(2) \simeq \operatorname{Gr}(2,4) \quad 14$

B Quantum K-theory for the dual pair $\operatorname{Gr}(2,5) \simeq \operatorname{Gr}(3,5) \quad 14$

\section{Introduction and outline}

The relation [1] between the Verlinde algebra [2] and quantum cohomology of Grassmannians [3-5] has been derived in refs. [6-8] by studying the small radius limit in an $S^{1}$-compactification of a $3 \mathrm{~d} \mathcal{N}=2$ supersymmetric gauge theory. This setup fits into the general framework of refs. $[9,10]$, which connects $3 \mathrm{~d}$ supersymmetric gauge theories to quantum K-theory, as opposed to quantum cohomology. An explicit 3d gauge theory/quantum K-theory correspondence between the $\mathcal{N}=2$ supersymmetric gauge theory and Givental's equivariant quantum K-theory [11-21] was proposed and studied in ref. [22]. For non-trivial Chern-Simons level, it involves the generalization to non-zero level defined in ref. [23]. A concrete relation between quantum K-theoretic correlators and the Verlinde numbers was proposed in [24].

In this note we carefully reconsider the relation between the Verlinde algebra, quantum cohomology and quantum K-theory of Grassmannians by studying the algebra of 3d Wilson line operators. The 3d Wilson loop algebra depends on the choice of Chern-Simons (CS) levels $\kappa$ and the matter spectrum. In a certain window for $\kappa$, the dimension of this $3 \mathrm{~d}$ algebra is fixed and equal to $\operatorname{dim}\left(H^{*}(\operatorname{Gr}(M, N))\right)$, but the quantum corrections to the structure constants of the ring of chiral operators associated with the loop algebra depend 
on the values of $\kappa$. The family of quantum algebras for different $\kappa$ contains the Verlinde algebra and quantum cohomology, the ordinary quantum K-theory studied by Buch and Mihalcea [25], and closely related algebras. The dependence on $\kappa$ drops out in the $2 \mathrm{~d}$ scaling limit, where the quantum algebras become isomorphic to quantum cohomology, as predicted by the arguments of refs. [1, 7].

\section{Generalized I-functions}

In the following we determine the Wilson line algebra by mapping Wilson line operators in a representation $R$ of the $3 \mathrm{~d} \mathcal{N}=2 \mathrm{U}(M)$ gauge theory to difference operators acting on Givental's $I$-function in quantum K-theory [11-21]. The $I$-function is the generating function of K-theoretic correlators with at least one insertion, defined as an Euler number on an appropriate compactification of the moduli space of maps from a curve $\Sigma$ to the target space $X$. The basic difference operators $p_{a}$ shift the exponentiated, complexified Kähler parameters $Q_{a}$ as $p_{a} Q_{a}=q Q_{a}, a=1, \ldots, h^{1,1}(X)$. Here $q$ is the weight for the twisting of the world-volume geometry $C \times{ }_{q} S^{1}$ of the $3 \mathrm{~d}$ theory, where the Riemann surface $C$ is topologically a two-sphere $S^{2}$ or a disk $D^{2}$. These twisted world-volume geometries relate to the Omega background of refs. [6, 26]. In Givental's formalism, $Q_{a}$ represent Novikov variables and $q$ is the weight for the $\mathbb{C}^{*}$ action in equivariant Gromov-Witten theory [27]. A relation between Wilson line operators and difference operators has been first studied in refs. $[6,28,29]$. A difference module structure in the variables $Q_{a}$ in quantum K-theory has been described in ref. [30]. For complete flag manifolds, the difference module had been related to the difference Toda lattice already in ref. [31]. Such a connection between gauge theory and integrable systems holds much more generally, as shown in the context of Bethe/gauge correspondence [10].

\subsection{Generalized $I$-functions of $\operatorname{Gr}(M, N)$}

The $3 \mathrm{~d}$ gauge theory/quantum K-theory correspondence of ref. [22] equates the $I$-function of permutation symmetric quantum K-theory of refs. [11-21] with the vortex sum of a supersymmetric partition function on $D^{2} \times_{q} S^{1}$. There are by now several methods to determine this partition function $[29,32-34]$. In this note we study the partition function for $3 \mathrm{~d} \mathcal{N}=2 \mathrm{U}(M)$ gauge theory with $N$ chiral matter multiplets in the fundamental representation, which realizes (for $N>M$ ) the complex $\operatorname{Grassmannian} \operatorname{Gr}(M, N)$ as its target space. The details of the computation of the partition function will be given elsewhere [35]; the result is ${ }^{1}$

$$
I_{\mathrm{Gr}(M, N)}^{S Q K}=c_{0} \sum_{0 \leq d_{a} \in \mathbb{Z}}(-Q)^{\sum \tilde{d}_{a}} q^{C S(\tilde{d})} \frac{\prod_{b>a}^{M} q^{\frac{1}{2} \tilde{d}_{a b}^{2}}\left(q^{\frac{1}{2} \tilde{d}_{a b}}-q^{-\frac{1}{2} \tilde{d}_{a b}}\right)}{\prod_{\alpha=1}^{N} \prod_{a=1}^{M} \prod_{r=1}^{d_{a}}\left(1-y_{\alpha} q^{r-\epsilon_{a}}\right)} .
$$

Here $c_{0}$ is a normalization factor chosen such that $\left.I_{\mathrm{Gr}(M, N)}^{S Q K}\right|_{Q=0}=(1-q)$. The parameters $y_{\alpha}$ arise as real masses of the $N$ matter multiplets and define the equivariant $I$-function

\footnotetext{
${ }^{1}$ To simplify some of the following formulas, a sign factor $(-1)^{M}$ has been absorbed in the definition of $Q$ relative to the conventions used in the mathematical literature.
} 
with respect to global flavor symmetry. Moreover $\tilde{d}_{a b}=\tilde{d}_{a}-\tilde{d}_{b}$ and $\tilde{d}_{a}=d_{a}-\epsilon_{a}$, where the parameters $\epsilon_{a}$ are related to the Chern roots $x_{a}$ of the dual $S^{*}$ of the tautological $\mathrm{U}(M)$ bundle $S$ over $\operatorname{Gr}(M, N)$ by $q^{\epsilon_{a}}=e^{\beta x_{a}}$. Here $\beta$ is the radius of $S^{1}$, which will be set to $\beta=1$ in most of the following. For vanishing real masses $y_{\alpha}=1$, the $I$-function takes values in the topological K-group $K(\operatorname{Gr}(M, N))$ - abbreviated in the following by $K(\mathrm{Gr}) .^{2}$ Using the Chern isomorphism, a basis of $\operatorname{dim}(K(\mathrm{Gr}))=\left(\begin{array}{l}N \\ M\end{array}\right)$ elements is provided by the Schur polynomials $\sigma_{\mu}\left(x^{K}\right)$ in the variables $x_{a}^{K}=1-e^{-x_{a}}$, where $\mu$ is a Young tableau in the set of tableaus fitting in a $M \times(N-M)$ box $\mathcal{B}_{N}$. The expansion

$$
I_{\mathrm{Gr}(M, N)}^{S Q K}=\sum_{\mu \in \mathcal{B}_{N}} I_{\mu}(q, Q) \sigma_{\mu}\left(x^{K}\right),
$$

represents the overlap of the groundstate of the $3 \mathrm{~d}$ theory with boundary states defined by a $3 \mathrm{~d}$ generalization of B-branes $[22,36]$. The choice of the variables $x_{a}^{K}$ is motivated by the $2 \mathrm{~d}$ limit $\beta \rightarrow 0$, where the basis $\left\{\sigma_{\mu}\left(x^{K}\right)\right\}$ reduces to the cohomological basis dual to the Schubert cycles, $\sigma_{\mu}\left(x^{K}\right) \stackrel{\beta \rightarrow 0}{\longrightarrow} \beta^{|\mu|}\left(\sigma_{\mu}(x)+O(\beta)\right) .^{3}$

The effective CS levels in the $3 \mathrm{~d}$ gauge theory are encoded in the term ${ }^{4}$

$$
q^{C S(\tilde{d})}=q^{\frac{1}{2} \hat{\kappa}_{S} \operatorname{tr}_{\mathrm{SU}(M)}\left(\tilde{d}^{2}\right)+\frac{1}{2} \hat{\kappa}_{A} \operatorname{tr}_{\mathrm{U}(1)}\left(\tilde{d}^{2}\right)+\hat{\kappa}_{R} \operatorname{tr}_{R}(\tilde{d})} .
$$

The parameters $\hat{\kappa}_{S}$ and $\hat{\kappa}_{A}$ specify the levels for the $\mathrm{SU}(M)$ and $\mathrm{U}(1)$ subgroups of the gauge group $\mathrm{U}(M)$, respectively, while $\hat{\kappa}_{R}$ is a level for the mixed gauge/R-symmetry CS term. The effective CS levels $\hat{\kappa}_{i}$ fulfill ${ }^{5}$

$$
\hat{\kappa}_{S} \in \mathbb{Z}, \quad \frac{\hat{\kappa}_{S}-\hat{\kappa}_{A}}{M} \in \mathbb{Z}, \quad 2 \hat{\kappa}_{R} \in \mathbb{Z},
$$

and the relation to the bare bulk CS terms $\kappa_{i}$ in the Lagrangian are [35]

$$
\hat{\kappa}_{S}=\kappa_{S}-M+\frac{N}{2}, \quad \hat{\kappa}_{A}=\kappa_{A}+\frac{N}{2}, \quad \hat{\kappa}_{R}=\kappa_{R}+\frac{N}{4} .
$$

Following the arguments of ref. [22], the level zero quantum K-theory of refs. [11-21] corresponds to zero effective CS levels $\hat{\kappa}_{i}=0$. For other levels $\hat{\kappa}_{i}$, one obtains a three parameter family of generalized $I$-functions. One can check that on the one-parameter slices $\left(\hat{\kappa}_{S}, \hat{\kappa}_{A}, \hat{\kappa}_{R}\right)=\left(\ell_{\square}, \ell_{\square},-\ell_{\square} / 2\right)$ and $\left(\hat{\kappa}_{S}, \hat{\kappa}_{A}, \hat{\kappa}_{R}\right)=\left(0, M \ell_{\operatorname{det}},-\ell_{\operatorname{det}} / 2\right)$ the above result respectively reproduces the $I$-functions at level $\ell_{\square}$ in the fundamental representation of $\mathrm{U}(M)$ and at level $\ell_{\text {det }}$ in the determinantal representation of $\mathrm{U}(M)$ in quantum K-theory with level structure, as derived in ref. [38] (see also ref. [24]). ${ }^{6}$

\footnotetext{
${ }^{2}$ More precisely, the $I$-function takes values in the equivariant K-group, and we obtain the K-group $K(\operatorname{Gr}(M, N))$ in the non-equivariant limit $y_{\alpha}=1$. In the following we mostly consider the nonequivariant limit.

${ }^{3}$ The connection of the K-theory basis $\left\{\sigma_{\mu}\left(x^{K}\right)\right\}$ to the basis of Grothendieck polynomials defined in ref. [37] will be discussed in section 4 .

${ }^{4}$ For a matrix $\sigma=\operatorname{diag}\left(\sigma_{1}, \ldots, \sigma_{M}\right)$ the trace symbols are defined $\operatorname{as~}_{\operatorname{tr}_{\mathrm{U}(M)}}\left(\sigma^{2}\right)=\sum_{a} \sigma_{a}^{2}, \operatorname{tr}_{\mathrm{U}(1)}\left(\sigma^{2}\right)=$ $\frac{1}{M}\left(\sum_{a} \sigma_{a}\right)^{2}, \operatorname{tr}_{\mathrm{SU}(M)}\left(\sigma^{2}\right)=\operatorname{tr}_{\mathrm{U}(M)}\left(\sigma^{2}\right)-\operatorname{tr}_{\mathrm{U}(1)}\left(\sigma^{2}\right)$ and $\operatorname{tr}_{R}(\sigma)=\sum_{a} \sigma_{a}$.

${ }^{5}$ The quantization relation linking $\hat{\kappa}_{S}$ and $\hat{\kappa}_{A}$ is a consequence of the global structure of the Lie group $\mathrm{U}(M) \simeq(\mathrm{SU}(M) \times \mathrm{U}(1)) / \mathbb{Z}_{M}$.

${ }^{6}$ Different K-theoretic I-functions for complex Grassmannians have also appeared in refs. [39, 40], however we did not find a suitable choice of CS terms (2.5) such that these match with the field theory result (2.1).
} 
To study the action of Wilson line operators in a representation $R$ of $\mathrm{U}(M)$ we will also need an abelianized version of the path integral for the maximal torus $\mathrm{U}(1)^{M} \subset \mathrm{U}(M){ }^{7}$ To this end we consider a modified sum $\hat{I}\left(Q_{a}\right)$ depending on $M$ weights $Q_{a}$,

$$
\hat{I}\left(Q_{a}\right)=c_{0} \sum_{\vec{d} \in \mathbb{Z}_{\geq 0}^{M}} c_{\vec{d}}(-Q)^{\vec{d}}, \quad(-Q)^{\vec{d}}=\prod_{a}\left(-Q_{a}\right)^{\tilde{d}_{a}},
$$

obtained from $I_{\operatorname{Gr}(M, N)}^{S Q K}(Q)$ by the replacement $(-Q)^{\sum d_{a}} \rightarrow(-Q)^{\vec{d}}$. The sum $\hat{I}$ satisfies the relation:

$$
\hat{I}=\Delta \tilde{I}, \quad \Delta=\prod_{b>a}\left(p_{a}-p_{b}\right),
$$

which can be viewed as a K-theoretic version of the Hori-Vafa formula [41]. Here $p_{a}=q^{\theta_{a}}$, $\theta_{a}=Q_{a} \partial_{Q_{a}}$, and

$$
\tilde{I}\left(Q_{a}\right)=c_{0} \sum_{\vec{d} \in \mathbb{Z}_{\geq 0}^{M}} q^{\gamma \sum_{b>a} \tilde{d}_{a} \tilde{d}_{b}} \prod_{a} I_{\mathbb{P}^{N-1}}^{d_{a}, \epsilon_{a}, \alpha, \beta}\left(-Q_{a}\right) .
$$

The constants are

$$
\alpha=\hat{\kappa}_{S}+\Delta_{\kappa}+M, \quad \beta=\hat{\kappa}_{R}-\frac{1}{2}(M-1), \quad \gamma=\frac{\hat{\kappa}_{A}-\hat{\kappa}_{S}}{M}-1=\Delta_{\kappa},
$$

with $\Delta_{\kappa}=\frac{\kappa_{A}-\kappa_{S}}{M}$. The last factor in eq. (2.8) involves the coefficients of a generalized $I$-function $I_{\mathbb{P}^{N-1}}^{\alpha, \beta}=\sum_{d \geq 0} I_{\mathbb{P}^{N-1}}^{d, \epsilon, \alpha, \beta}(Q)$ for $\mathbb{P}^{N-1}$ obtained from a $\mathrm{U}(1)$ gauge theory with CS levels $(\alpha, \beta)$, which reads

$$
I_{\mathbb{P}^{N-1}}^{d, \epsilon, \alpha, \beta}(Q)=\frac{Q^{\tilde{d}} q^{\frac{\alpha}{2} \tilde{d}^{2}+\beta \tilde{d}}}{\prod_{r=1}^{d}\left(1-q^{r-\epsilon}\right)^{N}} .
$$

The cross terms $\sim q^{\gamma} \tilde{d}_{a} \tilde{d}_{b}$ in eq. (2.8) obstruct a complete factorization into a product of $M \mathbb{P}^{N-1}$ factors, except for $\Delta_{\kappa}=0$. This condition is however incompatible with the canonical levels $\hat{\kappa}_{i}=0$ relevant for the level zero quantum K-theory.

\subsection{A window for the Chern-Simons levels}

The 3d gauge theory/quantum K-theory correspondence of ref. [22] equates the vortex sum of the $3 \mathrm{~d}$ gauge theory with the $I$-function for the permutation symmetric K-theory of refs. [11-21, 23]. That is, the $I$-function $I_{\operatorname{Gr}(N, M)}^{S Q K}(\delta t)$ in eq. (2.1) has in general a non-zero permutation symmetric input $\delta t$, determined by the expansion

$$
I_{\mathrm{Gr}(M, N)}^{S Q K}(\delta t)=(1-q)+\delta t+I_{\text {corr }}(\delta t), \quad \delta t \in \mathcal{K}_{+}, \quad I_{\text {corr }} \in \mathcal{K}_{-},
$$

which separates the perturbations $\delta t$ from the correlator part $I_{\text {corr }}(\delta t)$. Here $\mathcal{K}=K(\mathrm{Gr}) \otimes$ $\mathbb{C}\left(q, q^{-1}\right) \otimes \mathbb{C}[[Q]]$ is the symplectic loop space, and $\mathcal{K}_{ \pm}$the Lagrangian subspaces defined as $[23,30,43]$

$$
\begin{aligned}
& \mathcal{K}_{+}=K(\mathrm{Gr}) \otimes \mathbb{C}\left[q, q^{-1}\right] \otimes \mathbb{C}[[Q]], \\
& \mathcal{K}_{-}=K(\mathrm{Gr}) \otimes\{r(q) \in R(q) \mid r(0) \neq \infty \text { and } r(\infty)=0\} \otimes \mathbb{C}[[Q]],
\end{aligned}
$$

\footnotetext{
${ }^{7}$ Abelianization of Grassmannian sigma models has been used many times before in physics and mathematics; for some literature closely related to the present context see refs. [5, 38, 41, 42].
} 
where $R(q)$ denotes the field of rational functions in the variable $q$. The permutation symmetric input corresponds to a $3 \mathrm{~d}$ partition function perturbed by multi-trace operators [22]. To compute the chiral ring one wants to study the family of $3 \mathrm{~d}$ gauge theories perturbed only by single trace operators, corresponding to the ordinary quantum K-theory. Hence, one has to shift the multi-trace perturbations to zero, and subsequently perturb in the single trace directions, $I^{S Q K}(\delta t) \rightarrow I^{S Q K}(0)=I^{Q K}(0) \rightarrow I^{Q K}\left(t_{\text {single trace }}\right) .^{8}$

There is a certain window of CS levels, for which the $I$-function (2.1) is already at zero input, $\delta t=0$, and coincides with the unperturbed $I$-function $I_{\operatorname{Gr}(M, N)}^{Q K}(0)$ of ordinary quantum K-theory. From the definition of $\mathcal{K}_{+}$one sees that this requires that for any degree $\vec{d}$, the numerator of a summand of $\tilde{I}$ in eq. (2.8) contains no negative powers of $q$, and no positive power higher or equal than the degree of the denominator in $q$. Inspection of eq. (2.10) shows that this requires the CS levels to lie in the window

$$
H_{\kappa}: \quad\left|\kappa_{S}+\Delta_{\kappa}\right| \leq \frac{N}{2} .
$$

As discussed in the next section, the same condition assures that the $\mathbb{Z}[[Q]]$-module of Wilson loops has $\left(\begin{array}{l}N \\ M\end{array}\right)$ generators - in the following referred to as the dimension of the Wilson loop algebra - which is the number of generators of the K-theory group $K(\mathrm{Gr})$. There are extra operators outside the window $H_{\kappa}$.

\section{Algebra of difference operators}

\subsection{Wilson loops}

In the $3 \mathrm{~d}$ gauge theory one can consider more generally expectation values $\left\langle W_{R}\right\rangle$ of Wilson loop operators $W_{R}=\operatorname{tr}_{R} \mathrm{P} \exp \left(i \int_{S^{1}}\left(A_{\theta}-i \sigma\right) d \theta\right)$ wrapping $S^{1}$, where $\sigma$ is the real scalar field in the $3 \mathrm{~d}$ vector multiplet and $R$ is a representation of the gauge group. ${ }^{9}$ The insertion of a charge minus one Wilson line in the $a$-th $\mathrm{U}(1)$ factor in the path integral produces an extra factor $q^{\tilde{d}_{a}}$ in the sector with vortex number $d_{a}$. The vacuum expectation value of a $\mathrm{U}(1)^{M}$ Wilson line $W_{\vec{n}}=\prod_{a} W_{a}^{n_{a}}$ is then (up to a normalization factor) equal to a vortex sum

$$
\hat{I}_{\vec{n}}=c_{0} \Delta \sum_{\vec{d} \in \mathbb{Z}_{\geq 0}^{M}}(-Q)^{\vec{d}_{\tilde{c}}} \prod_{\vec{d}} \prod_{a} q^{\tilde{d}_{a} n_{a}},
$$

where $\Delta$ is the operator defined in eq. (2.7) and $\tilde{c}_{\vec{d}}$ are the coefficients of the $I$-function $\tilde{I}=c_{0} \sum_{d_{a} \geq 0}(-Q)^{\vec{d}_{\tilde{c}}} \tilde{d}_{\vec{d}}$ defined in eq. (2.8).

The extra factor $\prod_{a} q^{\tilde{d}_{a} n_{a}}$ can be generated by acting with a difference operator $\hat{I}$. Defining $\mathfrak{D}_{\vec{n}}(p)=\prod_{a} p_{a}^{n_{a}}$, with $p_{a}=q^{\theta_{a}}, \theta_{a}=Q_{a} \partial_{Q_{a}}$, one has

$$
\hat{I}_{\vec{n}}=\mathfrak{D}_{\vec{n}}(p) \Delta \tilde{I}
$$

Classically, a product of two Wilson line operators $W_{\vec{n}}$ and $W_{\vec{m}}$ corresponds to the insertion of $\prod_{a} q^{\tilde{d}_{a}\left(m_{a}+n_{a}\right)}$ in the sum, or the action with $\mathfrak{D}_{\vec{n}} \cdot \mathfrak{D}_{\vec{m}}=\mathfrak{D}_{\vec{m}+\vec{n}}$ on $\hat{I}$. In the quantum

\footnotetext{
${ }^{8}$ For more details, we refer to the discussion in refs. [30, 43] and [22].

${ }^{9}$ See e.g. refs. $[7,8,33,36,44]$ for studies of Wilson loops in Chern-Simons matter theories.
} 
theory, there are non-trivial relations between the Wilson lines for different $\vec{n}$. To this end, one notices that the sum $\tilde{I}$ satisfies the following difference equations:

$$
\begin{aligned}
\mathfrak{L}_{a} \tilde{I} & =O\left(\epsilon_{a}^{N}\right), \quad a=1, \ldots, M, \\
\mathfrak{L}_{a} & =\left(1-p_{a}\right)^{N}+Q_{a} q^{\frac{\alpha}{2}+\beta} p_{a}^{\alpha} \prod_{b \neq a} p_{b}^{\gamma} .
\end{aligned}
$$

These equations are inherited from the difference equations of the $\mathbb{P}^{N-1}$ factors, taking into account the additional CS terms. The $O\left(\epsilon_{a}^{N}\right)$ terms are set to zero in $H^{*}(G r(M, N))$. The difference equations allow to replace a difference operator $p_{a}^{D}$ acting on $\tilde{I}$ by an operator of lower degree in $p_{a}$. Here $D$ is the difference between the maximal degree and the (possibly negative) minimal degree in $p_{a}$ of the operator $\mathfrak{L}_{a}$ :

$$
D= \begin{cases}N & \text { for }\left|\kappa_{S}+\Delta_{\kappa}\right| \leq \frac{N}{2}, \\ \left|\kappa_{S}+\Delta_{\kappa}\right|+\frac{N}{2} & \text { for }\left|\kappa_{S}+\Delta_{\kappa}\right|>\frac{N}{2} .\end{cases}
$$

This reduces the algebra of difference operators acting on $\tilde{I}$ to $\mathfrak{D}_{\vec{n}}\left(p_{a}\right) /\left\langle\mathfrak{L}_{a}\right\rangle$ in terms of the ideal $\left\langle\mathfrak{L}_{a}\right\rangle$ generated by the operators $\mathfrak{L}_{a}$. Taking into account the extra factor $\Delta$ in the definition (2.7), it is necessary to further divide out by those relations that lie in the ideal $\left\langle\mathfrak{L}_{a}\right\rangle$ upon multiplying by $\Delta$. Thus, the algebra of difference operators acting on $\hat{I}$ is a $\mathbb{Z}[[Q]]$-module spanned by $\mathfrak{D}_{\vec{n}}\left(p_{a}\right) / \mathcal{I}$ with the ideal $\mathcal{I}$ generated by operators $\mathfrak{O}$, for which the products $\Delta \mathfrak{O}$ lies in the ideal $\left\langle\mathfrak{L}_{a}\right\rangle$.

In view of the relations (3.3), it is useful to also define shifted Wilson line operators $\hat{W}_{a}=1-W_{a}$, corresponding to the action of the difference operator $\delta_{a}=1-p_{a}$. In the $\mathrm{U}(M)$ theory, one has to restrict to permutation symmetric combinations of $\mathrm{U}(1)^{M}$ Wilson lines, which can be represented by Schur polynomials $\sigma_{\mu}$ (labeled by Young tableaus $\mu$ ) either in the operators $\delta_{a}$ or the operators $p_{a}$, i.e.,

$$
W_{\mu} \rightsquigarrow \mathfrak{D}_{\mu}(p) \text { with } \mathfrak{D}_{\mu}=\sigma_{\mu}\left(p_{a}\right), \quad \hat{W}_{\mu} \rightsquigarrow \hat{\mathfrak{D}}_{\mu}(\delta) \text { with } \hat{\mathfrak{D}}_{\mu}=\sigma_{\mu}\left(\delta_{a}\right) .
$$

For explicitness we focus now on the operators $\hat{\mathfrak{D}}_{\mu}$, which satisfy the algebra

$$
\hat{\mathfrak{D}}_{\mu} * \hat{\mathfrak{D}}_{\nu}=\sum_{\lambda} C_{\mu \nu}^{\lambda} \hat{\mathfrak{D}}_{\lambda}
$$

where the structure constants $C_{\mu \nu}^{\lambda}$ are the Littlewood-Richardson coefficients.

In the following we focus on the situation where $\mathfrak{L}_{a} \sim p_{a}^{D}+\ldots$ where the terms '.. ' are of non-negative homogenous degree smaller than $D$ in all operators $p_{b}$, which implies further constrains on $\gamma$. Since the factor $\Delta$ has degree $M-1$ with respect to each individual operator $p_{a}$, the relevant $\mathbb{Z}[[Q]]$-module of difference operators is generated by the operators $\hat{W}_{\lambda}$ for representations $\lambda$ fitting into a $M \times(D-M)$ box $\mathcal{B}_{D}$. Classically the ideal of relations is $\left\langle\hat{W}_{\lambda}\right\rangle=0$ for representations $\lambda$ not fitting into $\mathcal{B}_{D}$. This yields the number of representation-theoretic independent Wilson lines $\hat{W}_{\lambda}$, which is given by

$$
\left(\begin{array}{c}
D \\
M
\end{array}\right)=\operatorname{tr}(-)^{F}
$$


As indicated, this number is expected to coincide with the 3d Witten index, which has been computed already in refs. $[45,46]$ for $\Delta_{\kappa}=0$. Moreover, the UV computation in the GLSM can be shown to agree with the IR computation along the lines of ref. [47] for other values of $\Delta_{\kappa}[35]$.

After the reduction by the ideal (3.3), the structure constants depend on $Q, q$ and the CS levels, namely

$$
\hat{\mathfrak{D}}_{\mu} * \hat{\mathfrak{D}}_{\nu}=\sum_{\lambda \in \mathcal{B}_{D}} c_{\mu \nu}^{\lambda}\left(Q, q, \kappa_{i}\right) \hat{\mathfrak{D}}_{\lambda}
$$

Here the structure constants $c_{\mu \nu}^{\lambda}\left(Q, q, \kappa_{i}\right)$ are obtained from the action of the $\mathfrak{D}_{\mu}$ on $\hat{I}$ and taking the $Q_{a}=Q$ limit. One has $\left.c_{\mu \nu}^{\lambda}\right|_{Q=0}=C_{\mu \nu}^{\lambda}$.

To extract the Wilson loop algebra from the algebra of difference operators, one has to substract the terms that arise from the subsequent action of a difference operator on $Q$-dependent terms generated by the use of the relations (3.3) in a previous step. Since

$$
\delta(f(Q) \hat{I})=(\delta f) \hat{I}+f(Q q) \delta \hat{I}=f(Q) \delta \hat{I}+O(1-q)
$$

these terms are of higher order in an expansion of eq. (3.8) in a power series in $(1-q)$, and can be dropped by setting $q=1 .{ }^{10}$ The algebra of Wilson line operators is therefore given by the coefficient of $(1-q)^{0}$ :

$$
\hat{W}_{\mu} * \hat{W}_{\nu}=\sum_{\lambda \in \mathcal{B}_{D}} C_{\mu \nu}^{\lambda}\left(Q, \kappa_{i}\right) \hat{W}_{\lambda}
$$

with $C_{\mu \nu}^{\lambda}\left(Q, \kappa_{i}\right)=\left.c_{\mu \nu}^{\lambda}\left(Q, q, \kappa_{i}\right)\right|_{q=1}$.

The equations (3.3) are related to the vacuum equations of ref. [10], by first replacing $q^{\theta_{a}}$ by a commuting variable $p_{a}$ and subsequently taking the $q \rightarrow 1$ limit. The discussion is also similar to the treatment in ref. [7], where the algebra of Wilson loops has been computed from an ideal of relations for a $S^{3}$-partition function. ${ }^{11}$ It has been argued there, that the algebra should be independent of the manifold, on which the $3 \mathrm{~d}$ theory is compactified. A comparison of the above results with those in ref. [7] will be made below.

\subsection{Difference and differential equations for the $I$-function}

As discussed around eq. (2.13), the permutation symmetric $I$-function (2.1) coincides with the unperturbed $I$-function of the ordinary quantum K-theory inside the level window $H_{\kappa}$. The latter can be perturbed by operators $\Phi_{i} \in K(\mathrm{Gr})$, introducing a dependence on $\left(\begin{array}{l}N \\ M\end{array}\right)$ additional variables $t_{i}$. The correlators of the perturbed theory are captured by the

\footnotetext{
${ }^{10}$ There is no need to actually take the small radius limit $q \rightarrow 1$ here, since the algebra is independent of q. As explained around eq. (3.14), the $I$-function is related to the flat sections of the connections defined in eq. (3.13) and the above procedure amounts to keeping only the product on the r.h.s. .

${ }^{11}$ See also refs. [45, 46].
} 
Givental $J$-function ${ }^{12}$

$$
J(t)=(1-q)+t+\sum_{\beta, i, n \geq 0} \frac{Q^{\beta}}{n !}\left\langle\frac{\Phi_{i}}{1-q L}, t^{n}\right\rangle_{n+1, \beta} \Phi^{i} .
$$

The sum over $\beta$ runs over the degrees of rational curves and $n$ counts the number of insertions of the perturbations $t=\sum_{i} t_{i} \Phi_{i} \in \mathcal{K}_{+}$, where $\left\{\Phi_{i}\right\}, i=0, \ldots,\left(\begin{array}{l}N \\ M\end{array}\right)-1$ is a basis of $K(\mathrm{Gr})$. The elements $\Phi^{i}=\sum \chi^{i j} \Phi_{j}$ of the dual basis are defined with respect to the inner product

$$
\left(\Phi_{i}, \Phi_{j}\right)=\int_{\operatorname{Gr}(M, N)} \operatorname{td}(\operatorname{Gr}(M, N)) \Phi_{i} \Phi_{j}=: \chi_{i j} .
$$

The correlator part lies in $\mathcal{K}_{-}$and is defined as a holomorphic Euler characteristic on the moduli space of stable maps [48, 49].

The $J$-function of $\operatorname{Gr}(M, N)$ relates to a fundamental solution $T \in \operatorname{End}(K(\mathrm{Gr}))$ of the flatness equations $[48,50]$

$$
\begin{aligned}
(1-q) \partial_{t_{i}} T & =T \Phi_{i^{*}}, & & i=0, \ldots,\left(\begin{array}{c}
N \\
M
\end{array}\right)-1, \\
q^{\theta} T & =T A q^{\theta}, & \theta & =Q \partial_{Q},
\end{aligned}
$$

where $*$ denotes the quantum product, $A \in \operatorname{End}(K(\mathrm{Gr}))$ and we suppress the $Q, t, q$ dependence of the endomorphisms. In terms of $T$ one has

$$
J(t)=(1-q) T \Phi_{0}=(1-q) \sum_{i}\left(T \Phi_{0}, \Phi_{i}\right) \Phi^{i},
$$

where $\Phi_{0}=1$ denotes the unit. The systems of first order equations (3.13) imply differential equations for the $J$-function. For concreteness and simplicity we consider the small deformation space spanned by the element $\Phi_{1}=1-e^{c_{1}(S)}$ and suppress the index on $t$. Suppose there is a relation in the quantum K-theoretic algebra of the form

$$
\sum_{k=0}^{d} a_{k} \nabla^{k} \Phi_{0}=0, \quad \nabla=(1-q) \partial_{t}+\Phi_{1} *,
$$

for some integer $d \leq\left(\begin{array}{l}N \\ M\end{array}\right)$, where $a_{k}$ are some $(Q, q)$-dependent coefficients. Then

$$
0=\left(T \sum_{k} a_{k} \nabla^{k} \Phi_{0}, \Phi_{i}\right)=\sum_{k} a_{k} \partial_{t}^{k}\left(T \Phi_{0}, \Phi_{i}\right),
$$

where the second step uses that the basis elements $\Phi_{i}$ are constant. This implies the differential equation

$$
\sum_{k=0}^{d} a_{k} D^{k} J=0, \quad D=\partial_{t} .
$$

\footnotetext{
${ }^{12}$ The $I$-function studied before, and the $J$-function below, are in general connected by a non-trivial transformation, the $3 \mathrm{~d}$ mirror map. At zero perturbation, this transformation is trivial for $\operatorname{Gr}(M, N)$ with canonical CS levels, i.e., $J(0)=I(0)$. For non-zero level, the correlators and the inner product in the following two equations have to be defined as in ref. [23].
} 
Similarly, a relation $\sum_{k} b_{k}\left(A q^{\theta}\right)^{k} \Phi_{0}=0$ in the quantum algebra implies the difference equation

$$
\sum_{k} b_{k} p^{k} J=0, \quad p=q^{\theta}
$$

In this way, relations in the quantum K-theory algebra translate to quantum differential and difference equations for the $J$-function and vice versa.

\section{Selected examples}

\subsection{Quantum cohomology and factorized case}

Factorized case. The simplest subset of theories arises for the choice of CS levels with $\Delta_{\kappa}=0$, i.e., equal bare CS levels in the $\mathrm{SU}(N)$ and $\mathrm{U}(1)$ factors, but unequal effective levels, $\hat{\kappa}_{S} \neq \hat{\kappa}_{A}$. In this case, the relations in eq. (3.3) factorize into $M$ separate equations

$$
\delta_{a}^{N}=-Q_{a} q^{\frac{\alpha}{2}+\beta} p_{a}^{\alpha} .
$$

These quantum relations are equivalent to those obtained in ref. [7] from a $S^{3}$ partition function.

A K-theory algebra isomorphic to quantum cohomology/Verlinde algebra. In the window $\left|\kappa_{S}+\Delta_{\kappa}\right| \leq \frac{N}{2}$, the dimension of the Wilson line algebra equals $\operatorname{dim}(K(\mathrm{Gr}))=\left(\begin{array}{l}N \\ M\end{array}\right)$, and the algebra of Wilson lines can be isomorphic to the quantum cohomology ring of $\operatorname{Gr}(M, N)$, which, furthermore, is isomorphic to the Verlinde algebra of the gauged Wess-Zumino-Witten model $\mathrm{U}(M) / \mathrm{U}(M)$ at level $(N-M)$ [1]. This happens for the special case $\alpha=\beta=\gamma=0$, where the difference equation becomes

$$
\left(1-p_{a}\right)^{N}=-Q_{a}
$$

This is, up to our sign convention, the same relation as in quantum cohomology [3-5], and thus will give an isomorphic algebra after projecting to $\mathrm{U}(M)$. The operators generating these algebras are $3 \mathrm{~d}$ Wilson lines $\hat{W}_{\mu}$ in the quantum K-theoretic ring or the corresponding operators related to the cohomology classes $\left[\sigma_{\mu}\right] \in H^{*}(\operatorname{Gr}(M, N))$ in the $2 \mathrm{~d}$ case [8].

2d limit. In the $3 \mathrm{~d}$ gauge theory compactified on an $S^{1}$ of radius $\beta$, one can consider a simple small radius limit, in which the quantum K-theoretic $I$-function reduces to the cohomological $I$-function. To this end one sets $q=e^{-\hbar \beta}$, and rescales the operators and the FI parameter by factors of $\beta$ [22]. For any CS levels, the $2 d$ limit of the relations (3.3) is

$$
\left(\beta \theta_{a}\right)^{N}=-Q_{a}
$$

which is again the relation in quantum cohomology, after a renormalization $Q_{a} \rightarrow Q_{a} e^{N \ln \beta}$. The different K-theoretic algebras of Wilson loops distinguished by different CS levels therefore reduce all to the quantum cohomology algebra, in agreement with the arguments of refs. $[1,7]$. 


\subsection{Chiral ring at level zero}

The canonical case from the point of quantum K-theory is however at zero effective CS level, related the ordinary K-theory of refs. [48, 49]. In the following we study the chiral ring from the algebra of difference operators and show that the result agrees with the results in the mathematical literature on the quantum product of Schubert structure sheaves [25].

The canonical case lies inside the level window $H_{\kappa}$ if $N>M$, which is required for the theory to have a supersymmetric vacuum, see e.g., ref. [7]. The chiral ring can therefore be studied directly from the $I$-function (2.1) at zero input. The difference equations (3.3) take the form

$$
\delta_{a}^{N}=-Q_{a} \frac{p_{a}^{M}}{\prod_{b=1}^{M} p_{b}}, \quad a=1, \ldots, M .
$$

The appearance of a denominator on the r.h.s. is harmless, as the operators $p_{a}$ are invertible. Due to the non-factorized form, the general polynomial reduction defined by the ideal (4.4) is not entirely trivial, but straightforward to perform for given values of $M$ and $N$.

As an illustrative example we discuss the first non-trivial case $\operatorname{Gr}(2,4)$. Performing the polynomial reduction of the difference operators one obtains, up to order $O(1-q)$ terms, the multiplication table

$$
\begin{array}{ll}
\sigma_{1} * \sigma_{1}=\sigma_{2}+\sigma_{1,1}, & \sigma_{2} * \sigma_{2,2}=Q\left(\sigma_{1,1}+\sigma_{2,1}-\sigma_{2,2}\right)+Q^{2}, \\
\sigma_{1} * \sigma_{2}=\sigma_{2,1}+Q\left(2+\rho_{1}\right), & \sigma_{1,1} * \sigma_{1,1}=\sigma_{2,2}, \\
\sigma_{1} * \sigma_{1,1}=\sigma_{2,1}, & \sigma_{1,1} * \sigma_{2,1}=Q \rho_{1}, \\
\sigma_{1} * \sigma_{2,1}=\sigma_{2,2}+Q\left(1+\rho_{1}\right), & \sigma_{1,1} * \sigma_{2,2}=Q\left(\sigma_{2}-\sigma_{2,1}\right), \\
\sigma_{1} * \sigma_{2,2}=Q \rho_{1}, & \sigma_{2,1} * \sigma_{2,1}=Q\left(\sigma_{2}+\sigma_{1,1}-\sigma_{2,2}\right)+Q^{2}, \\
\sigma_{2} * \sigma_{2}=\sigma_{2,2}+Q\left(\sigma_{1}+\rho_{2}\right)+Q^{2}, & \sigma_{2,1} * \sigma_{2,2}=Q\left(\sigma_{2,1}-\sigma_{2,2}\right)+Q^{2}, \\
\sigma_{2} * \sigma_{1,1}=Q\left(1+\rho_{1}\right), & \sigma_{2,2} * \sigma_{2,2}=Q^{2} . \\
\sigma_{2} * \sigma_{2,1}=Q \rho_{2}+Q^{2}, &
\end{array}
$$

Here $\sigma_{\mu}$ stands for either $\hat{W}_{\mu}$ or $\hat{\mathfrak{D}}_{\mu}(\delta)$ and we used the abbreviations $\rho_{1}=\sigma_{1}+\sigma_{2}-\sigma_{1,1}-$ $\sigma_{2,1}, \rho_{2}=\sigma_{1}+\sigma_{2}+\sigma_{1,1}-\sigma_{2,2}$.

In the classical limit $Q=0$, the difference operators $\sigma_{\mu}(\delta)$ become Schur polynomials $\sigma_{\mu}\left(x_{a}^{K}\right)$ in the variables $x_{a}^{K}=1-e^{-x_{a}}$. The polynomials $\sigma_{\mu}\left(x^{K}\right)$ represent Chern characters of a basis $\left\{\phi_{\mu}\right\}$ for $K(\mathrm{Gr})$. The pairing $\chi\left(\phi_{\mu}, \phi_{\nu}\right)$ on the basis $\left\{\phi_{\mu}\right\}$ has already determinant one, but is non-minimal in the sense that the entries are not all 0 or 1 . The polynomials $\sigma_{\mu}\left(x^{K}\right)$ are related by a linear transformation of determinant one to the Grothendieck polynomials $\mathcal{O}_{\mu}\left(x^{K}\right)$ of ref. [37]:

$$
\begin{aligned}
\mathcal{O}_{1} & =\sigma_{1}-\sigma_{1,1}, & \mathcal{O}_{2} & =\sigma_{2}-\sigma_{2,1}, \quad \mathcal{O}_{1,1}=\sigma_{1,1}, \\
\mathcal{O}_{2,1} & =\sigma_{2,1}-\sigma_{2,2}, & \mathcal{O}_{2,2} & =\sigma_{2,2} .
\end{aligned}
$$

The inner product (3.12) on the new basis is

$$
\chi\left(\mathcal{O}_{\mu}, \mathcal{O}_{\nu}\right)=\left(\begin{array}{llllll}
1 & 1 & 1 & 1 & 1 & 1 \\
1 & 1 & 1 & 1 & 1 & 0 \\
1 & 1 & 1 & 0 & 0 & 0 \\
1 & 1 & 0 & 1 & 0 & 0 \\
1 & 1 & 0 & 0 & 0 & 0 \\
1 & 0 & 0 & 0 & 0 & 0
\end{array}\right), \quad \operatorname{det}(\chi)=1
$$


The $\mathcal{O}_{\mu}$ are the Chern characters of the structure sheaves of the Schubert cycles. After the basis change, we obtain for the quantum multiplication of the structure sheaves ${ }^{13}$

\begin{tabular}{c|ccccc}
$*$ & $\mathcal{O}_{1}$ & $\mathcal{O}_{2}$ & $\mathcal{O}_{1,1}$ & $\mathcal{O}_{2,1}$ & $\mathcal{O}_{2,2}$ \\
\hline $\mathcal{O}_{1}$ & $\mathcal{O}_{2}+\mathcal{O}_{1,1}-\mathcal{O}_{2,1}$ & - & - & - & - \\
$\mathcal{O}_{2}$ & $\mathcal{O}_{2,1}$ & $\mathcal{O}_{2,2}$ & - & - & - \\
$\mathcal{O}_{1,1}$ & $\mathcal{O}_{2,1}$ & $Q$ & $\mathcal{O}_{2,2}$ & - & - \\
$\mathcal{O}_{2,1}$ & $\mathcal{O}_{2,2}+Q\left(1-\mathcal{O}_{1}\right)$ & $Q \mathcal{O}_{1}$ & $Q \mathcal{O}_{1}$ & $Q\left(\mathcal{O}_{2}+\mathcal{O}_{1,1}-\mathcal{O}_{2,1}\right)$ & - \\
$\mathcal{O}_{2,2}$ & $Q \mathcal{O}_{1}$ & $Q \mathcal{O}_{1,1}$ & $Q \mathcal{O}_{2}$ & $Q \mathcal{O}_{2,1}$ & $Q^{2}$
\end{tabular}

These multiplications agree with the result of ref. [25], which has been obtained by quite different methods.

At zero deformations $t=0$, as detailed in ref. [35], the K-theoretic quantum product can be also obtained from the critical locus of the 1-loop effective $\mathcal{N}=2$ twisted superpotential $\widetilde{W}[10]$. In the example, there are two relations obtained from $e^{d \widetilde{W}}=1$, which can be written as

$$
r_{1}=\mathcal{O}_{1}^{3}-2 \mathcal{O}_{1} \mathcal{O}_{1,1}+\mathcal{O}_{1}^{2} \mathcal{O}_{1,1}, \quad r_{2}=\mathcal{O}_{1}^{2} \mathcal{O}_{1,1}-\mathcal{O}_{1,1}^{2}+\mathcal{O}_{1} \mathcal{O}_{1,1}^{2}-Q
$$

One can check that, similiar to the cohomological case [3-5], the K-theoretic quantum product (4.8) is isomorphic to a polynomial ring in two generators $\mathcal{O}_{1}, \mathcal{O}_{1,1}$, divided by the ideal of relations $r_{a}=0$

$$
\mathbb{Z}\left[\mathcal{O}_{1}, \mathcal{O}_{1,1}, Q\right] /\left(r_{1}, r_{2}\right) .
$$

The Grassmannians $\operatorname{Gr}(M, N)$ and $\operatorname{Gr}(N-M, N)$ are related by the well-known classical geometric duality, and this duality persists at the level of quantum K-theory. Under the duality a Young tableau is exchanged with its transposed Young tableau. In the self-dual example $\operatorname{Gr}(2,4)$, the duality in the quantum theory is reflected in the symmetry of the quantum multiplication (4.8) under the exchange of $\mathcal{O}_{2}$ and $\mathcal{O}_{1,1}$. In the appendix B we discuss the less trivial example of $\operatorname{Gr}(2,5) \simeq \operatorname{Gr}(3,5)$ of dual quantum K-theories and its relation to Seiberg like dualities of ref. [51].

\subsection{Difference equations at level zero}

To determine the difference equations satisfied by $I_{\mathrm{Gr}(M, N)}(0)$ in (2.1), it suffices to know the quantum product at zero deformation computed above. It is straightforward to show, that at $t=0$, the action of $A$ in eq. (3.13) is equal to the quantum product with $\Phi_{1}$, i.e. $\left.A\right|_{t=0}=\left.\left(\Phi_{1} *\right)\right|_{t=0}$. Computing the linear relations between suitable powers of $\left(A q^{\theta}\right)^{k} \Phi_{0}$, we find the difference operators annihilating the $I$-function $I_{\operatorname{Gr}(M, N)}(0)$ listed in table 1 . The difference operators for $\operatorname{Gr}(2,5)$ and $\operatorname{Gr}(2,6)$ are of degree 4 and 8 in $Q$ and the complete expressions are lengthy, see e.g. eq. (B.5). On the r.h.s. we have included the $2 \mathrm{~d}$ limit of the difference operator described around eq. (4.3), which gives a differential operator in $\tilde{Q}$. Here $\tilde{Q}=Q \beta^{-N}$ is the renormalized parameter with $\beta$ the $S^{1}$ radius. These limits agree with the differential operators of ref. [52] that annihilate the cohomological $I$-function.

\footnotetext{
${ }^{13}$ Since the multiplication is commutative, the entries above the diagonal are omitted, as indicated by the dashes.
} 


\begin{tabular}{|r|l|l|}
\hline$(M, N):$ & $3 \mathrm{~d}$ difference operator & $2 \mathrm{~d}$ limit \\
\hline$(2,3):$ & $\delta^{3}-Q$ & $\theta^{3}-\tilde{Q}$ \\
$(2,4):$ & $\delta^{5}+Q(p q+1)\left(p^{2} q-1\right)$ & $\theta^{5}-2(2 \theta+1) \tilde{Q}$ \\
$(3,4):$ & $\delta^{4}+Q$ & $\theta^{4}+\tilde{Q}$ \\
$(2,5):$ & $\delta^{7}(-1+\delta+q)^{3}+O(Q)$ & $(\theta-1)^{3} \theta^{7}-\left(11 \theta^{2}+11 \theta+3\right) \theta^{3} \tilde{Q}-\tilde{Q}^{2}$ \\
$(2,6):$ & $\delta^{9}(\delta+q-1)^{5}$ & $(\theta-2)\left[-\theta^{5}(1+2 \theta)\left(4+13 \theta+13 \theta^{2}\right) \tilde{Q}\right.$ \\
& $\left(\delta+q^{2}-1\right)+O(Q)$ & $\left.+(-1+\theta)^{5} \theta^{9}-3(2+3 \theta)(4+3 \theta) \tilde{Q}^{2}\right]$ \\
\hline
\end{tabular}

Table 1. Difference operators for low values of $(M, N)$ and their $2 \mathrm{~d}$ limits.

\subsection{Perturbed theory for $\operatorname{Gr}(2,4)$}

The above results for the $I$-function and the chiral ring hold at zero perturbation $t=0$. Methods to reconstruct the $I$-function at non-zero $t$ from the $I$-function at $t=0$ have been described in refs. [18, 43, 50], using the difference module structure in quantum K-theory, first established in ref. [30] for the ordinary theory.

These reconstruction theorems use the difference operators (3.13) to deform the $I$ function and are therefore limited to the directions in $K(X)$ generated by repeated action of $A$ on the unit $\Phi_{0}$. In the case of the Grassmannian $\operatorname{Gr}(M, N)$, this reconstructs the subspace of the deformations $t=\sum_{i} t_{i}\left(\mathcal{O}_{1}\right)^{i}$ generated by the difference operator associated with the line bundle $e^{c_{1}(S)}=1-\mathcal{O}_{1}$.

From the view point of the $3 \mathrm{~d}$ gauge theory, the full deformation space can be accessed by adding single trace deformations in any representation of the gauge group [22], and this is not limited to the subspace generated by powers of $\mathcal{O}_{1}$. It suffices to know the action of Wilson line operators $\hat{W}_{\mu}$ on the $I$-function for any representation $\mu$. This action has been already reconstructed in section 3 in terms of the difference operators $\hat{\mathfrak{D}}_{\mu}$ acting on the generalized $I$-function $\hat{I}\left(Q_{a}\right)$ depending on $M$ Novikov variables $Q_{a}$, and setting $Q_{a}=Q$ at the end.

The general reconstruction for $\operatorname{Gr}(M, N)$ will be discussed in ref. [35]. Here we briefly discuss the simplest case of the level zero quantum K-theory for $\operatorname{Gr}(2,4)$ at non-zero $t$, where one can use duality to reconstruct the $I$-function from the reconstruction methods of refs. $[43,50]$. Classically, the Grassmannian $\operatorname{Gr}(2,4)$ can be described via the Plücker embedding as a quadratic hypersurface $\mathbb{P}^{5}[2]$ in $\mathbb{P}^{5}$. One can verify that this relation extends to a duality of the quantum K-theory at $t=0$, by establishing the equality of the level zero $I$-functions

$$
I_{\mathrm{Gr}(2,4)}(0) \simeq I_{\mathbb{P}^{5}[2]}(0)=(1-q) \sum_{d \geq 0} Q^{\tilde{d}} \frac{\prod_{r=1}^{2 d}\left(1-q^{r-2 \epsilon}\right)}{\prod_{r=1}^{d}\left(1-q^{r-\epsilon}\right)^{6}} .
$$

The easiest way to show this equation is to check that the r.h.s. satisfies the same difference equation from table 1 and to compare the first terms in the $Q$-expansion to fix the normalization. 
For the hypersurface in $\mathbb{P}^{5}$, we choose the basis $\Phi_{k}=\left(1-e^{-H}\right)^{k}, k=0, \ldots, 4$, where $H$ denotes the hyperplane class. By the classical duality it is related to the basis $\left\{\mathcal{O}_{\mu}\right\}$ for $K(\operatorname{Gr}(2,4))$ by $\Phi_{k}=\left(\mathcal{O}_{1}\right)^{k}$. Because of the symmetry exchanging $\mathcal{O}_{2}$ and $\mathcal{O}_{1,1}$ at $t=0$, one can reduce the six dimensional basis $\left\{\mathcal{O}_{\mu}\right\}$ to the five-dimensional basis $\left\{\left(\mathcal{O}_{1}\right)^{k}\right\}$, as long as the direction $\mathcal{O}_{2}-\mathcal{O}_{1,1}$ stays undeformed. The structure constants at $t=0$ in the basis $\left\{\left(\mathcal{O}_{1}\right)^{k}\right\}$ are

$$
C_{\mathcal{O}_{1},\left\{\mathcal{O}_{\mu}\right\}}=\left(\begin{array}{cccccc}
0 & 1 & 0 & 0 & 0 & 0 \\
0 & 0 & 1 & 1 & -1 & 0 \\
0 & 0 & 0 & 0 & 1 & 0 \\
0 & 0 & 0 & 0 & 1 & 0 \\
Q & -Q & 0 & 0 & 0 & 1 \\
0 & Q & 0 & 0 & 0 & 0
\end{array}\right) \rightarrow C_{\Phi_{1},\left\{\Phi_{k}\right\}}=\left(\begin{array}{ccccc}
0 & 1 & 0 & 0 & 0 \\
0 & 0 & 1 & 0 & 0 \\
-Q & Q & 0 & 1 & 0 \\
2 Q & -3 Q & 0 & 0 & 1 \\
0 & 2 Q & 0 & 0 & 0
\end{array}\right) .
$$

One can check that the r.h.s. agrees with the structure constants at $t=0$ computed from $I_{\mathbb{P}^{5}[2]}(0)$.

One way to reconstruct the perturbed theory is to integrate the flatness equations (3.13), as described in ref. [50]. This has the advantage of getting easily all order expressions in the general perturbation ${ }^{14} T=\sum_{k=1}^{4} T_{k} \Phi_{k}$ at fixed power of $Q$. Restricting to the direction $t=T_{1}$, which is the integrable deformation in the $2 \mathrm{~d}$ limit, the perturbed structure constants at order $Q^{1}$ obtained in this way are

$$
\left.C_{\Phi_{1}}(t)\right|_{Q^{1}}=Q e^{t}\left(\begin{array}{ccccc}
0 & 0 & 0 & 0 & 0 \\
0 & 0 & 0 & 0 & 0 \\
-1 & (t+1) & -\frac{1}{2} t(t+2) & \frac{1}{6} t^{2}(t+3) & -\frac{1}{24} t^{3}(t+4) \\
2 & -(2 t+3) & t(t+3) & -\frac{1}{6} t^{2}(2 t+9) & \frac{1}{12} t^{3}(t+6) \\
0 & 2 & -2 t & t^{2} & -\frac{1}{3} t^{3}
\end{array}\right) .
$$

In the perturbed theory, the structure constants get contributions also from higher order in $Q$. Some higher order terms are given in eq. (A.1).

\section{Acknowledgments}

We would like to thank Tudor Dimofte, Heeyeon Kim, Nikita Nekrasov, Yongbin Ruan, Eric Sharpe, Chris Woodward, and Ming Zhang for discussions and correspondences. The work of P.M. is supported by the German Excellence Cluster Origins. U.N. is supported by the graduate school BCGS, and A.T. is supported by the DFG.

\footnotetext{
${ }^{14}$ The dependence on $T_{0}$ is fixed by the string equation $[17,49]$.
} 


\section{A Perturbed structure constants for $\mathbb{P}^{5}(2) \simeq \operatorname{Gr}(2,4)$}

The perturbed structure constants (4.13) in the direction $t \Phi_{1}$ up to order $Q^{3}$ are:

$$
\begin{aligned}
& Q^{0}:\left(\begin{array}{lllll}
0 & 1 & 0 & 0 & 0 \\
0 & 0 & 1 & 0 & 0 \\
0 & 0 & 0 & 1 & 0 \\
0 & 0 & 0 & 0 & 1 \\
0 & 0 & 0 & 0 & 0
\end{array}\right) \\
& Q^{1}:\left(\begin{array}{ccccc}
0 & 0 & 0 & 0 & 0 \\
0 & 0 & 0 & 0 & 0 \\
\frac{1}{6}\left(-t^{3}-3 t^{2}-6 t-6\right) & \frac{2 t^{3}}{3}+\frac{3 t^{2}}{2}+2 t+1 & -\frac{1}{2} t\left(2 t^{2}+3 t+2\right) & \frac{1}{6} t^{2}(4 t+3) & -\frac{t^{3}}{6} \\
\frac{t^{3}}{3}+t^{2}+2 t+2 & -\frac{3 t^{3}}{2}-\frac{7 t^{2}}{2}-5 t-3 & \frac{1}{2} t\left(5 t^{2}+8 t+6\right) & -\frac{1}{6} t^{2}(11 t+9) & \frac{t^{3}}{2} \\
0 & \frac{t^{3}}{3}+t^{2}+2 t+2 & -t\left(t^{2}+2 t+2\right) & t^{2}(t+1) & -\frac{t^{3}}{3}
\end{array}\right)+O\left(t^{4}\right), \\
& Q^{2}:\left(\begin{array}{ccccc}
0 & 0 & 0 & 0 & 0 \\
\frac{t^{4}}{24} & -\frac{t^{4}}{12} & \frac{t^{4}}{24} & 0 & 0 \\
-\frac{1}{24} t^{2}\left(53 t^{2}+36 t+12\right) & \frac{1}{24} t^{2}\left(113 t^{2}+56 t+12\right) & -\frac{2}{3} t^{3}(4 t+1) & \frac{5 t^{4}}{12} & 0 \\
\frac{1}{12} t^{2}\left(71 t^{2}+52 t+18\right) & -\frac{1}{24} t^{2}\left(309 t^{2}+164 t+36\right) & \frac{1}{2} t^{3}(15 t+4) & -\frac{5 t^{4}}{4} & 0 \\
-\frac{1}{3} t^{2}\left(10 t^{2}+8 t+3\right) & \frac{1}{12} t^{2}\left(91 t^{2}+52 t+12\right) & -\frac{2}{3} t^{3}(7 t+2) & \frac{5 t^{4}}{6} & 0
\end{array}\right)+O\left(t^{5}\right) \\
& Q^{3}:\left(\begin{array}{ccccc}
0 & 0 & 0 & 0 & 0 \\
0 & 0 & 0 & 0 & 0 \\
-\frac{5 t^{4}}{12} & \frac{5 t^{4}}{12} & 0 & 0 & 0 \\
\frac{5 t^{4}}{4} & -\frac{5 t^{4}}{4} & 0 & 0 & 0 \\
-\frac{5 t^{4}}{6} & \frac{5 t^{4}}{6} & 0 & 0 & 0
\end{array}\right)+O\left(t^{5}\right)
\end{aligned}
$$

\section{B Quantum K-theory for the dual pair $\operatorname{Gr}(2,5) \simeq \operatorname{Gr}(3,5)$}

The Grassmannians $\operatorname{Gr}(2,5)$ and $\operatorname{Gr}(3,5)$ are related by the well-known classical geometric duality. In the following we compute the quantum K-theoretic product and test the duality at the quantum level.

The quantum product for the K-theoretic Schur polynomials $\sigma_{\mu}\left(x^{K}\right)$ in $\operatorname{Gr}(2,5)$ can be derived from the difference operators eq. (4.4) along the lines of section 3. The K-theoretic Schur polynomials $\sigma_{\mu}\left(x^{K}\right)$ are related to the Grothedieck polynomials $\mathcal{O}_{\mu}\left(x^{K}\right)$ for $\operatorname{Gr}(2,5)$ by

$$
\begin{array}{llll}
\mathcal{O}_{1}=\sigma_{1}-\sigma_{1,1}, & \mathcal{O}_{2}=\sigma_{2}-\sigma_{2,1}, & \mathcal{O}_{1,1}=\sigma_{1,1}, \\
\mathcal{O}_{3}=\sigma_{3}-\sigma_{3,1}, & \mathcal{O}_{2,1}=\sigma_{2,1}-\sigma_{2,2}, & \mathcal{O}_{3,1}=\sigma_{3,1}-\sigma_{3,2}, \\
\mathcal{O}_{2,2}=\sigma_{2,2}, & \mathcal{O}_{3,2}=\sigma_{3,2}-\sigma_{3,3}, & \mathcal{O}_{3,3}=\sigma_{3,3} .
\end{array}
$$


In the basis $\left\{\mathcal{O}_{\mu}\right\}$ with the given ordering, the inner product (3.12) is

$$
\chi\left(\mathcal{O}_{\mu}, \mathcal{O}_{\nu}\right)=\left(\begin{array}{llllllllll}
1 & 1 & 1 & 1 & 1 & 1 & 1 & 1 & 1 & 1 \\
1 & 1 & 1 & 1 & 1 & 1 & 1 & 1 & 1 & 0 \\
1 & 1 & 1 & 1 & 1 & 1 & 1 & 0 & 0 & 0 \\
1 & 1 & 1 & 1 & 0 & 1 & 0 & 1 & 0 & 0 \\
1 & 1 & 1 & 0 & 1 & 0 & 0 & 0 & 0 & 0 \\
1 & 1 & 1 & 1 & 0 & 1 & 0 & 0 & 0 & 0 \\
1 & 1 & 1 & 0 & 0 & 0 & 0 & 0 & 0 & 0 \\
1 & 1 & 0 & 1 & 0 & 0 & 0 & 0 & 0 & 0 \\
1 & 1 & 0 & 0 & 0 & 0 & 0 & 0 & 0 & 0 \\
1 & 0 & 0 & 0 & 0 & 0 & 0 & 0 & 0 & 0
\end{array}\right), \quad \operatorname{det}(\chi)=1
$$

Written in the basis of the structure sheaves, the multiplication table becomes

\begin{tabular}{c|cccc}
$*$ & $\mathcal{O}_{1}$ & $\mathcal{O}_{2}$ & $\mathcal{O}_{1,1}$ & $\mathcal{O}_{3}$ \\
\hline $\mathcal{O}_{1}$ & $\mathcal{O}_{1,1}-\mathcal{O}_{2,1}+\mathcal{O}_{2}$ & - & - & - \\
$\mathcal{O}_{2}$ & $\mathcal{O}_{2,1}-\mathcal{O}_{3,1}+\mathcal{O}_{3}$ & $\mathcal{O}_{2,2}+\mathcal{O}_{3,1}-\mathcal{O}_{3,2}$ & - & - \\
$\mathcal{O}_{1,1}$ & $\mathcal{O}_{2,1}$ & $\mathcal{O}_{3,1}$ & $\mathcal{O}_{2,2}$ & - \\
$\mathcal{O}_{3}$ & $\mathcal{O}_{3,1}$ & $\mathcal{O}_{3,2}$ & $Q$ & $\mathcal{O}_{3,3}$ \\
$\mathcal{O}_{2,1}$ & $\mathcal{O}_{2,2}+\mathcal{O}_{3,1}-\mathcal{O}_{3,2}$ & $\mathcal{O}_{3,2}+Q\left(1-\mathcal{O}_{1}\right)$ & $\mathcal{O}_{3,2}$ & $Q \mathcal{O}_{1}$ \\
$\mathcal{O}_{3,1}$ & $\mathcal{O}_{3,2}+Q\left(1-\mathcal{O}_{1}\right)$ & $\mathcal{O}_{3,3}+Q\left(\mathcal{O}_{1}-\mathcal{O}_{2}\right)$ & $Q \mathcal{O}_{1}$ & $Q \mathcal{O}_{2}$ \\
$\mathcal{O}_{2,2}$ & $\mathcal{O}_{3,2}$ & $Q \mathcal{O}_{1}$ & $\mathcal{O}_{3,3}$ & $Q \mathcal{O}_{1,1}$ \\
$\mathcal{O}_{3,2}$ & $\mathcal{O}_{3,3}+Q\left(\mathcal{O}_{1}-\mathcal{O}_{2}\right)$ & $Q\left(\mathcal{O}_{1,1}-\mathcal{O}_{2,1}+\mathcal{O}_{2}\right)$ & $Q \mathcal{O}_{2}$ & $Q \mathcal{O}_{2,1}$ \\
$\mathcal{O}_{3,3}$ & $Q \mathcal{O}_{2}$ & $Q \mathcal{O}_{2,1}$ & $Q \mathcal{O}_{3}$ & $Q \mathcal{O}_{2,2}$
\end{tabular}

\begin{tabular}{c|ccccc}
$*$ & $\mathcal{O}_{2,1}$ & $\mathcal{O}_{3,1}$ & $\mathcal{O}_{2,2}$ & $\mathcal{O}_{3,2}$ & $\mathcal{O}_{3,3}$ \\
\hline $\mathcal{O}_{1}$ & - & - & - & - & - \\
$\mathcal{O}_{2}$ & - & - & - & - & - \\
$\mathcal{O}_{1,1}$ & - & - & - & - & - \\
$\mathcal{O}_{3}$ & - & - & - & - & - \\
$\mathcal{O}_{2,1}$ & $\mathcal{O}_{3,3}+Q\left(\mathcal{O}_{1}-\mathcal{O}_{2}\right)$ & - & - & - & - \\
$\mathcal{O}_{3,1}$ & $Q\left(\mathcal{O}_{1,1}-\mathcal{O}_{2,1}+\mathcal{O}_{2}\right)$ & $Q\left(\mathcal{O}_{2,1}-\mathcal{O}_{3,1}+\mathcal{O}_{3}\right)$ & - & - & - \\
$\mathcal{O}_{2,2}$ & $Q \mathcal{O}_{2}$ & $Q \mathcal{O}_{2,1}$ & $Q \mathcal{O}_{3}$ & - & - \\
$\mathcal{O}_{3,2}$ & $Q\left(\mathcal{O}_{2,1}-\mathcal{O}_{3,1}+\mathcal{O}_{3}\right)$ & $Q\left(\mathcal{O}_{2,2}+\mathcal{O}_{3,1}-\mathcal{O}_{3,2}\right)$ & $Q \mathcal{O}_{3,1}$ & $Q \mathcal{O}_{3,2}+Q^{2}$ & $\left(1-\mathcal{O}_{1}\right)$ \\
$\mathcal{O}_{3,3}$ & $Q \mathcal{O}_{3,1}$ & $Q_{3,2}$ & $Q^{2}$ & $Q^{2} \mathcal{O}_{1}$ & $Q^{2} \mathcal{O}_{1,1}$
\end{tabular}

The above multiplications agree with those derived from the quantum Pieri and Giambelli rules of ref. [25]. Moreover, the above quantum product can be shown to be isomorphic to the polynomial ring

$$
\begin{aligned}
\mathbb{Z}\left[\mathcal{O}_{1}, \mathcal{O}_{1,1}, Q\right] /\left(r_{1}, r_{2}\right) & \\
r_{1} & =\mathcal{O}_{1}^{4}+2 \mathcal{O}_{1}^{3} \mathcal{O}_{1,1}+\mathcal{O}_{1}^{2} \mathcal{O}_{1,1}^{2}-3 \mathcal{O}_{1}^{2} \mathcal{O}_{1,1}-2 \mathcal{O}_{1} \mathcal{O}_{1,1}^{2}+\mathcal{O}_{1,1}^{2} \\
r_{2} & =\mathcal{O}_{1}^{3} \mathcal{O}_{1,1}+2 \mathcal{O}_{1}^{2} \mathcal{O}_{1,1}^{2}+\mathcal{O}_{1} \mathcal{O}_{1,1}^{3}-2 \mathcal{O}_{1} \mathcal{O}_{1,1}^{2}-\mathcal{O}_{1,1}^{3}-Q
\end{aligned}
$$


The product determines the difference operator $\mathfrak{L}$ annihilating the $I$-function as discussed in section 4.3. Writing $\mathfrak{L}=\sum_{i \geq 0} Q^{i} \mathfrak{L}_{i}$ one finds the operator of degree $\left(\begin{array}{l}5 \\ 2\end{array}\right)=10$ in $\delta$ and degree 4 in $Q$ :

$$
\begin{aligned}
\mathfrak{L}_{0}= & -25 \delta^{7}\left(q^{2}+3 q+1\right)^{3}(\delta+q-1)^{3}, \\
\mathfrak{L}_{1}= & 25 \delta^{10}(q-1) q^{4}(q+1)\left(q^{2}+q+1\right)-5 \delta^{6} q^{4}\left(q^{2}+3 q+1\right)^{3}\left(63 q^{2}+10 q+2\right) \\
& +5 \delta^{5} q^{4}\left(q^{2}+3 q+1\right)^{3}\left(78 q^{2}-16 q-7\right)-25 \delta^{4}(q-1) q^{4}(9 q+2)\left(q^{2}+3 q+1\right)^{3} \\
& +25 \delta^{3}(q-1)^{2} q^{3}(2 q+1)\left(q^{2}+3 q+1\right)^{3}+5 \delta^{9}(q-1) q^{4}\left(2 q^{4}+2 q^{3}-3 q^{2}-13 q-13\right) \\
& -5 \delta^{8}(q-1) q^{4}\left(24 q^{5}+47 q^{4}+32 q^{3}+12 q^{2}-3 q-12\right) \\
& +5 \delta^{7} q^{4}\left(25 q^{8}+191 q^{7}+677 q^{6}+1087 q^{5}+815 q^{4}+295 q^{3}+39 q^{2}-7 q+3\right), \\
\mathfrak{L}_{2}= & \delta^{10} q^{9}-5 \delta^{8}(q-1) q^{9}(q+1)+25 q^{8}\left(q^{2}+3 q+1\right)^{2}\left(q^{4}+3 q^{2}+1\right) \\
& -5 \delta^{7}(q-1) q^{9}\left(25 q^{4}+50 q^{3}+51 q^{2}+28 q+3\right)+5 \delta^{6}(q-1) q^{9}\left(115 q^{4}+190 q^{3}+162 q^{2}+79 q+4\right) \\
& -25 \delta q^{8}\left(q^{2}+3 q+1\right)\left(5 q^{6}+18 q^{5}+19 q^{4}+45 q^{3}+21 q^{2}+12 q+5\right) \\
& -\delta^{5} q^{8}\left(25 q^{8}+225 q^{7}+1699 q^{6}+875 q^{5}+205 q^{4}-5 q^{3}-125 q^{2}+201 q+25\right) \\
& +5 \delta^{4} q^{8}\left(25 q^{8}+210 q^{7}+766 q^{6}+598 q^{5}+685 q^{4}+525 q^{3}+200 q^{2}+104 q+12\right) \\
& -5 \delta^{3} q^{8}\left(50 q^{8}+390 q^{7}+1096 q^{6}+1193 q^{5}+1645 q^{4}+1190 q^{3}+475 q^{2}+179 q+32\right) \\
& +25 \delta^{2} q^{8}\left(10 q^{8}+72 q^{7}+180 q^{6}+240 q^{5}+345 q^{4}+240 q^{3}+108 q^{2}+45 q+10\right), \\
\mathfrak{L}_{3}= & -5 \delta^{7} q^{15}+5 \delta^{6}(5 q-2) q^{14}+\delta^{5}\left(25 q^{5}+25 q^{4}-70 q^{2}+34 q-25\right) q^{13} \\
& -5 \delta^{4}(q-1)\left(25 q^{4}+48 q^{3}+48 q^{2}+21 q+25\right) q^{13}+5 \delta^{3}(q-1)\left(50 q^{4}+92 q^{3}+93 q^{2}+61 q+50\right) q^{13} \\
& -5 \delta^{2}(q-1)\left(50 q^{4}+88 q^{3}+91 q^{2}+70 q+50\right) q^{13}+5 \delta(q-1)\left(25 q^{4}+42 q^{3}+45 q^{2}+38 q+25\right) q^{13} \\
& -5(q-1)\left(5 q^{4}+8 q^{3}+9 q^{2}+8 q+5\right) q^{13}, \\
\mathfrak{L}_{4}= & \delta^{5} q^{19}-5 \delta^{4} q^{19}+10 \delta^{3} q^{19}-10 \delta^{2} q^{19}+5 \delta q^{19}-q^{19} .
\end{aligned}
$$

The computation for the dual case $\operatorname{Gr}(3,5)$ is similar. The relation of structure sheaves $\mathcal{O}_{\mu}\left(x^{K}\right)$ and the Schur basis $\sigma_{\mu}\left(x^{K}\right)$ is now

$$
\begin{array}{lll}
\mathcal{O}_{1}=\sigma_{1}-\sigma_{1,1}+\sigma_{1,1,1}, & \mathcal{O}_{2}=\sigma_{2}-\sigma_{2,1}+\sigma_{2,1,1}, \\
\mathcal{O}_{1,1}=\sigma_{1,1}-2 \sigma_{1,1,1}, & \mathcal{O}_{2,1}=\sigma_{2,1}-\sigma_{2,2}-2 \sigma_{2,1,1}+2 \sigma_{2,2,1}-\sigma_{2,2,2}, \\
\mathcal{O}_{1,1,1}=\sigma_{1,1,1}, & \mathcal{O}_{2,2}=\sigma_{2,2}-2 \sigma_{2,2,1}+\sigma_{2,2,2}, \\
\mathcal{O}_{2,1,1}=\sigma_{2,1,1}-\sigma_{2,2,1}+\sigma_{2,2,2}, & \mathcal{O}_{2,2,1}=\sigma_{2,2,1}-2 \sigma_{2,2,2}, \quad \mathcal{O}_{2,2,2}=\sigma_{2,2,2} .
\end{array}
$$

The inner product on the basis of structure sheaves is

$$
\chi\left(\mathcal{O}_{\mu}, \mathcal{O}_{\nu}\right)=\left(\begin{array}{llllllllll}
1 & 1 & 1 & 1 & 1 & 1 & 1 & 1 & 1 & 1 \\
1 & 1 & 1 & 1 & 1 & 1 & 1 & 1 & 1 & 0 \\
1 & 1 & 1 & 1 & 1 & 0 & 1 & 0 & 0 & 0 \\
1 & 1 & 1 & 1 & 1 & 1 & 0 & 1 & 0 & 0 \\
1 & 1 & 1 & 1 & 1 & 0 & 0 & 0 & 0 & 0 \\
1 & 1 & 0 & 1 & 0 & 1 & 0 & 0 & 0 & 0 \\
1 & 1 & 1 & 0 & 0 & 0 & 0 & 0 & 0 & 0 \\
1 & 1 & 0 & 1 & 0 & 0 & 0 & 0 & 0 & 0 \\
1 & 1 & 0 & 0 & 0 & 0 & 0 & 0 & 0 & 0 \\
1 & 0 & 0 & 0 & 0 & 0 & 0 & 0 & 0 & 0
\end{array}\right), \quad \operatorname{det}(\chi)=1
$$

Taking acount a sign change $Q \rightarrow-Q$, which is due to our sign convention explained in footnote 1 , the quantum multiplication table for $\operatorname{Gr}(3,5)$ computed by polynomial reduction is equal to the one obtained from (B.3) by transposing Young tableaus labelling the 
Grothendieck polynomials, i.e., $\mathcal{O}_{\mu} \rightarrow \mathcal{O}_{\mu^{T}}$. Moreover, the difference operator for $\operatorname{Gr}(3,5)$ coincides with that in eq. (B.5), which implies that the expansion coefficients (2.2) of the $I$-functions agree up to linear combination.

From the point of the underlying 3d field theory, the agreement of the level zero quantum K-theory for $\operatorname{Gr}(2,5)$ and $\operatorname{Gr}(3,5)$ is expected in view of the Seiberg type dualities studied in ref. [51]. The agreement of the $I$-functions is the statement, that the $D^{2} \times S^{1}$ partition functions for these $3 \mathrm{~d}$ theories agree upon appropriate identification of the vacua on both sides. From the algebraic point, the isomorphism between the Wilson line algebras is less obvious, and the vacuum equations $e^{d \widetilde{W}}=1$ of the Bethe/gauge correspondence of ref. [10] are different in the two theories. Non-trivial equivalences of algebras of this type have been discussed ref. [7].

Open Access. This article is distributed under the terms of the Creative Commons Attribution License (CC-BY 4.0), which permits any use, distribution and reproduction in any medium, provided the original author(s) and source are credited.

\section{References}

[1] E. Witten, The Verlinde algebra and the cohomology of the Grassmannian, hep-th/9312104 [INSPIRE].

[2] E.P. Verlinde, Fusion Rules and Modular Transformations in 2D Conformal Field Theory, Nucl. Phys. B 300 (1988) 360 [InSPIRE].

[3] C. Vafa, Topological Landau-Ginzburg models, Mod. Phys. Lett. A 6 (1991) 337 [inSPIRE].

[4] K.A. Intriligator, Fusion residues, Mod. Phys. Lett. A 6 (1991) 3543 [hep-th/9108005] [INSPIRE].

[5] S. Cecotti and C. Vafa, On classification of $N=2$ supersymmetric theories, Commun. Math. Phys. 158 (1993) 569 [hep-th/9211097] [INSPIRE].

[6] N. Nekrasov, Four Dimensional Holomorphic Theories, Ph.D. Thesis, Princeton University (1996) [http://scgp.stonybrook.edu/people/faculty/bios/nikita-nekrasov].

[7] A. Kapustin and B. Willett, Wilson loops in supersymmetric Chern-Simons-matter theories and duality, arXiv:1302.2164 [INSPIRE].

[8] N. Nekrasov, BPS/CFT correspondence IV: $\sigma$-models and defects in gauge theory, Lett. Math. Phys. 109 (2019) 579 [arXiv:1711.11011] [INSPIRE].

[9] N.A. Nekrasov and S.L. Shatashvili, Quantization of Integrable Systems and Four Dimensional Gauge Theories, in 16th International Congress on Mathematical Physics, pp. 265-289 (2009) [DOI] [arXiv: 0908.4052] [INSPIRE].

[10] N.A. Nekrasov and S.L. Shatashvili, Supersymmetric vacua and Bethe ansatz, Nucl. Phys. B Proc. Suppl. 192-193 (2009) 91 [arXiv:0901.4744] [INSPIRE].

[11] A. Givental, Permutation-equivariant quantum K-theory I. Introduction. Elementary $K$-theory of $\bar{M}_{0, n} / S_{n}$, arXiv:1508.02690.

[12] A. Givental, Permutation-equivariant quantum K-theory II. Fixed point localization, arXiv: 1508.04374. 
[13] A. Givental, Permutation-equivariant quantum K-theory III. Lefschetz' fixed point formula on $\bar{M}_{0, n} / S_{n}$, arXiv: 1508.06697.

[14] A. Givental, Permutation-equivariant quantum K-theory IV. $D_{q}$-modules, arXiv: 1509.00830.

[15] A. Givental, Permutation-equivariant quantum K-theory V. Toric q-hypergeometric functions, arXiv:1509.03903.

[16] A. Givental, Permutation-equivariant quantum K-theory VI. Mirrors, arXiv:1509.07852.

[17] A. Givental, Permutation-equivariant quantum K-theory VII. General theory, arXiv: 1510.03076.

[18] A. Givental, Permutation-equivariant quantum K-theory VIII. Explicit reconstruction, arXiv: 1510.06116.

[19] A. Givental, Permutation-equivariant quantum K-theory IX. Quantum Hirzebruch-Riemann-Roch in all genera, arXiv:1709.03180.

[20] A. Givental, Permutation-equivariant quantum K-theory X. Quantum Hirzebruch-Riemann-Roch in genus 0, arXiv:1710.02376.

[21] A. Givental, Permutation-equivariant quantum k-theory XI. Quantum Adams-Riemann-Roch, arXiv: 1711.04201.

[22] H. Jockers and P. Mayr, A 3d Gauge Theory/Quantum k-theory Correspondence, Adv. Theor. Math. Phys. 24 (2020) 327 [arXiv: 1808.02040] [INSPIRE].

[23] Y. Ruan and M. Zhang, The level structure in quantum K-theory and mock theta functions, arXiv: 1804.06552.

[24] Y. Ruan and M. Zhang, Verlinde/Grassmannian Correspondence and Rank $2 \delta$-wall-crossing, arXiv: 1811.01377 [INSPIRE].

[25] A.S. Buch and L.C. Mihalcea, Quantum K-theory of Grassmannians, Duke Math. J. 156 (2011) 501 [arXiv:0810.0981].

[26] N. Nekrasov and E. Witten, The Omega Deformation, Branes, Integrability, and Liouville Theory, JHEP 09 (2010) 092 [arXiv: 1002.0888] [INSPIRE].

[27] A. Givental, Equivariant Gromov-Witten invariants, Int. Math. Res. Not. 1996 (1996) 613 [alg-geom/9603021].

[28] T. Dimofte, D. Gaiotto and S. Gukov, 3-Manifolds and 3d Indices, Adv. Theor. Math. Phys. 17 (2013) 975 [arXiv:1112.5179] [inSPIRE].

[29] C. Beem, T. Dimofte and S. Pasquetti, Holomorphic Blocks in Three Dimensions, JHEP 12 (2014) 177 [arXiv:1211.1986] [INSPIRE].

[30] A. Givental and V. Tonita, The Hirzebruch-Riemann-Roch theorem in true genus-0 quantum $k$-theory, arXiv:1106.3136 [INSPIRE].

[31] A. Givental and Y.-P. Lee, Quantum K-theory on flag manifolds, finite-difference Toda lattices and quantum groups, Invent. Math. 151 (2003) 193 [math/0108105].

[32] A. Gadde, S. Gukov and P. Putrov, Walls, Lines, and Spectral Dualities in 3d Gauge Theories, JHEP 05 (2014) 047 [arXiv: 1302.0015] [InSPIRE].

[33] Y. Yoshida and K. Sugiyama, Localization of $3 d \mathcal{N}=2$ Supersymmetric Theories on $S^{1} \times D^{2}$, arXiv:1409.6713 [INSPIRE]. 
[34] T. Dimofte, D. Gaiotto and N.M. Paquette, Dual boundary conditions in 3d SCFT's, JHEP 05 (2018) 060 [arXiv: 1712.07654] [INSPIRE].

[35] H. Jockers, P. Mayr, U. Ninad and A. Tabler, Wilson loop algebras and quantum K-theory for Grassmannians II, to appear.

[36] S. Cecotti, D. Gaiotto and C. Vafa, tt* geometry in 3 and 4 dimensions, JHEP 05 (2014) 055 [arXiv: 1312.1008] [INSPIRE].

[37] A. Lascoux and M.-P. Schützenberger, Structure de Hopf de l'anneau de cohomologie et de l'anneau de Grothendieck d'une variété de drapeaux, C.R. Acad. Sci. Paris Sér. I Math. 295 (1982) 629.

[38] Y. Wen, K-Theoretic I-function of $V / /{ }_{\theta} \mathbf{G}$ and Application, arXiv:1906.00775.

[39] K. Taipale, K-theoretic J-functions of type A flag varieties, arXiv:1110.3117 [INSPIRE].

[40] C. Woodward and G. Xu, An open quantum Kirwan map, arXiv:1806.06717 [INSPIRE].

[41] K. Hori and C. Vafa, Mirror symmetry, hep-th/0002222 [INSPIRE].

[42] S. Martin, Symplectic quotients by a nonAbelian group and by its maximal torus, math/0001002 [INSPIRE].

[43] A. Givental, Explicit reconstruction in quantum cohomology and K-theory, Ann. Fac. Sci. Toulouse Math. 25 (2016) 419 [arXiv:1506.06431].

[44] A. Kapustin, B. Willett and I. Yaakov, Exact Results for Wilson Loops in Superconformal Chern-Simons Theories with Matter, JHEP 03 (2010) 089 [arXiv:0909.4559] [INSPIRE].

[45] F. Benini and A. Zaffaroni, Supersymmetric partition functions on Riemann surfaces, Proc. Symp. Pure Math. 96 (2017) 13 [arXiv:1605.06120] [INSPIRE].

[46] C. Closset and H. Kim, Comments on twisted indices in $3 d$ supersymmetric gauge theories, JHEP 08 (2016) 059 [arXiv: 1605.06531] [INSPIRE].

[47] K. Intriligator and N. Seiberg, Aspects of $3 d N=2$ Chern-Simons-Matter Theories, JHEP 07 (2013) 079 [arXiv: 1305.1633] [inSPIRE].

[48] A. Givental, On the WDVV equation in quantum K-theory, Michigan Math. J. 48 (2000) 295 [math/0003158].

[49] Y.P. Lee, Quantum k-theory I: foundations, math/0105014 [INSPIRE].

[50] H. Iritani, T. Milanov and V. Tonita, Reconstruction and Convergence in Quantum $K$-Theory via Difference Equations, arXiv:1309.3750 [INSPIRE].

[51] F. Benini, C. Closset and S. Cremonesi, Comments on 3d Seiberg-like dualities, JHEP 10 (2011) 075 [arXiv: 1108.5373] [INSPIRE].

[52] V.V. Batyrev, I. Ciocan-Fontanine, B. Kim and D. van Straten, Conifold transitions and mirror symmetry for Calabi-Yau complete intersections in Grassmannians, Nucl. Phys. B 514 (1998) 640 [alg-geom/9710022] [INSPIRE]. 